\title{
Corrigendum: Translational Research Studies Unraveling the Origins of Psoriatic Arthritis: Moving Beyond Skin and Joints
}

\author{
Janne W. Bolt ${ }^{1,2,3}$, Chaja M. J. van Ansenwoude ${ }^{1,2,3}$, Ihsan Hammoura ${ }^{1,2,3}$, \\ Marleen G. van de Sande ${ }^{1,2,3}$ and Lisa G. M. van Baarsen ${ }^{1,2,3 *}$ \\ ${ }^{1}$ Department of Rheumatology \& Clinical Immunology, Amsterdam Institute for Infection \& Immunity, Amsterdam UMC, \\ University of Amsterdam, Amsterdam, Netherlands, ${ }^{2}$ Department of Experimental Immunology, Amsterdam Institute for \\ Infection \& Immunity, Amsterdam UMC, University of Amsterdam, Amsterdam, Netherlands, ${ }^{3}$ Amsterdam Rheumatology \& \\ Immunology Center (ARC), Academic Medical Center, Amsterdam, Netherlands
}

Keywords: early psoriatic arthritis, psoriasis, immunopathogenesis, translational, animal models

\section{OPEN ACCESS}

Approved by:

Frontiers Editorial Office,

Frontiers Media SA, Switzerland

*Correspondence:

Lisa G. M. van Baarsen

e.g.vanbaarsen@amsterdamumc.nl

Specialty section:

This article was submitted to

Rheumatology,

a section of the journal

Frontiers in Medicine

Received: 19 August 2021

Accepted: 20 August 2021

Published: 08 September 2021

Citation:

Bolt JW, van Ansenwoude CMJ, Hammoura I, van de Sande MG and van Baarsen LGM (2021)

Corrigendum: Translational Research

Studies Unraveling the Origins of

Psoriatic Arthritis: Moving Beyond Skin

and Joints. Front. Med. 8:761410.

doi: 10.3389/fmed.2021.761410

\section{A Corrigendum on}

Translational Research Studies Unraveling the Origins of Psoriatic Arthritis: Moving Beyond Skin and Joints

by Bolt, J. W., van Ansenwoude, C. M. J., Hammoura, I., van de Sande, M. G., and van Baarsen, L. G. M. (2021). Front. Med. 8:711823. doi: 10.3389/fmed.2021.711823

In the original article, the Funding statement was inadvertently absent. The statement should be:

"LB received funding from a ZonMw VIDI project (91718371). MS received funding from a ZonMw VENI project (09150161810112). LB and MS received funding from the European Union's Horizon 2020 research and innovation programme under the Marie Skłodowska-Curie grant agreement No 847551 (ARCAID)."

The authors apologize for this error and state that this does not change the scientific conclusions of the article in any way. The original article has been updated.

Publisher's Note: All claims expressed in this article are solely those of the authors and do not necessarily represent those of their affiliated organizations, or those of the publisher, the editors and the reviewers. Any product that may be evaluated in this article, or claim that may be made by its manufacturer, is not guaranteed or endorsed by the publisher.

Copyright () 2021 Bolt, van Ansenwoude, Hammoura, van de Sande and van Baarsen. This is an open-access article distributed under the terms of the Creative Commons Attribution License (CC BY). The use, distribution or reproduction in other forums is permitted, provided the original author(s) and the copyright owner(s) are credited and that the original publication in this journal is cited, in accordance with accepted academic practice. No use, distribution or reproduction is permitted which does not comply with these terms. 